\title{
A Correlative Study of Geomagnetic Storms Associated with Solar Wind and IMF Features During Solar Cycle 23
}

\author{
Balveer S. Rathore, Subhash C. Kaushik, K. A. Firoz, D. C. Gupta, A. K. Shrivastava, \\ Krishna Kant Parashar, and Ram Mohan Bhaduriya
}

\begin{abstract}
A geomagnetic storm is a global disturbance in Earth's magnetic field usually occurred due to abnormal conditions in the interplanetary magnetic field (IMF) and solar wind plasma emissions caused by various solar phenomenon. Furthermore the magnitude of these geomagnetic effects largely depend upon the configuration and strength of potentially geo-effective solar/interplanetary features. In the present study the identification of 220 geomagnetic storms associated with disturbance storm time (Dst) decrease of more than $\mathbf{- 5 0} \mathrm{nT}$ to $-300 \mathrm{nT}$, have been made, which are observed during 1996-2007, the time period spanning over solar cycle 23 . The study is made statistically between the Dst strength (used as an indicator of the geomagnetic activity) and the peak value obtained by solar wind plasma parameters and IMF $B$ as well as its components. We have used the hourly values of Dst index and the wind measurements taken by various satellites. Our results inferred that yearly occurrences of geomagnetic storms are strongly correlated with 11-year sunspot cycle. We observed that IMF B is highly geo-effective during the main phase of magnetic storms, while it more significant at the time of storm peak, which is further contributed by southward component of IMF Bz, substantiating earlier findings. The correlation between Dst and wind velocity is higher, as compared with IMF $B z$ and ion density. It has been verified that geomagnetic storm intensity is correlated well with the total magnetic field strength of IMF better than with its southward component.
\end{abstract}

Index Terms-Geomagnetic Storm, Interplanetary magnetic field (IMF), Disturbance storm time (Dst), Solar Cycle.

\section{INTRODUCTION}

Geo-magnetic storms generally occurred due to abnormal conditions in the interplanetary magnetic field (IMF) and solar wind plasma emissions caused by various solar phenomenon [1,2]. The study of these worldwide disturbances of Earth's magnetic field are important in understanding the dynamics of solar-terrestrial environment and furthermore because such storms can cause life threatening power outrages, satellite damage, communication failure and navigational problems [2-4]. Since the beginning

This work was supported by UGC, New Delhi through its Research Award Scheme to SCK, and independent projects to DCG and AKS, KAF's work is financed by KASI, South Korea.

Balveer S. Rathore, D.C. Gupta, A.K. Shrivastava, K.K. Paarashar and R.M. Bhaduriya are with the School of Studies in Physics, Jiwaji University, Gwalior, 474004, M.P. India. (e-mail: balveer_ra@yahoo.co.in).

Subhash C. Kaushik was with Department of Physics, Government Autonomous PG Excellence College, Datia. He is now with the School of Studies in Physics, Jiwaji University, Gwalior, 474004, M.P. India. (e-mail: subashkaushik@rediffmail.com;kaushik@prl.res.in)

K. A. Firoz, is with Solar and Space Weather Research Group, Korea Astronomy and Space Science Institute, Daejeon, 305-348, South Korea. (e-mail: kafiroz@kasi.re.kr ; kazifiroz2002@gmail.com) of the space age, the cause of geomagnetic activity has been sought in a number of correlative studies [1]. It is suggested that geomagnetic, activity is related to variety of interplanetary plasma/ field parameters, e.g. Solar wind velocity $\mathrm{V}$, interplanetary magnetic field (IMF) $\mathrm{B}$ and $\mathrm{Bz}[1$, $2,4]$. Furthermore, the strong geomagnetic disturbance is associated with passage of magnetic cloud [3, 4], which causes intense and sever geomagnetic storms $[5,6]$.

It is well established fact that solar wind is continuously emanating from the sun's outer corona and engulf the entire heliosphere. It mainly consists of hot electrons and protons flowing supersonically and caused due to extremely high coronal temperature helping ionized plasma to overcome the gravitation attraction of the Sun. The density and speed of this flow is highly variable and depends solely upon the conditions which has caused it to eject. The solar wind carries with it the magnetic field of Sun, which when enters to the interplanetary medium is termed as Interplanetary Magnetic Field (IMF). The strength and orientation of this magnetic field associated with solar wind depends up on its interaction between slow and fast solar wind originating from coronal holes and leads to create co-rotating interaction region (CIR) $[1,5,7]$.

Geomagnetic disturbance are generally represented by geomagnetic storms and sudden ionosphere disturbance (SIDs). These are caused by the disturbances originated at solar atmosphere, interplanetary (IP) shocks and / or stream interfaces associated with high speed solar wind streams (HSSWS) [8, 9]. These are associated with Coronal holes, which occur in Polar Regions or higher latitude. Fast CME produce transient IP shocks, which cause storm sudden commencement at earth. Geomagnetic storms are associated with isolated disappearing filaments $[3,10,11]$. The occurrence of prominences and flares are also associated with varying phases of sunspot cycle leading to the geomagnetic storms. The strength of IMF and its fluctuations have also shown to be most important parameter affecting the geomagnetic field condition. South direction of IMF, allows sufficient energy transfer from the solar wind into the Earth magnetosphere through magnetic reconnection [12-14].

As a geomagnetic storm lasts usually a few to several days in duration. However, sometimes the recovery phase of a geomagnetic storm lasts one to two weeks or even for longer durations. These kind of long-duration events were termed as High-Intensity Long-Duration Continuous AE Activity events (HILDCAA events). In a study of such events made by Tsurutani and Gonzalez (1987), it was suggested that continuous injections to the ring current take place during these events in such manner that the ring current does 
not, or cannot, decay rapidly [15-18]. Various studies have reported that these geo-effective events are further associated with CME's, solar flares, SEPs and also with other solar wind transients [19-22]. Which not only significantly produce the depressions in the earth's ring current but also modulates the cosmic ray intensity, causes Forbush decrease events, and produce ground level enhancement events too [23-24].

In this paper the statistical study has been performed to analyze these geomagnetic storms recorded by various geomagnetic observatories identified with the help of Disturbance storm time index (Dst). This Dst index is taken as an indicator of geomagnetically disturbed condition, as it represents the depressions in the ring current as a result of its interaction with the plasma signatures having their roots originated at solar surface or from some other exotic environment. We investigated various solar parameters/ interplanetary magnetic field components which were potentially geo- effective and occurred during the solar activity period of solar cycle- 23 .

\section{SElection CRITERIA AND DATA}

The disturbances in the geomagnetic field are caused by fluctuation in the solar wind impinging on the earth. The disturbances may be limited to the high-latitude polar region, unless the interplanetary magnetic field (IMF) carried by the solar wind has long periods (several hours or more) of southward component $(\mathrm{Bz}<0)$ with large magnitudes [ 2-4]. The occurrence of such a period stresses the magnetosphere continuously, causing the magnetic field disturbance to reach the equatorial region. The degree of the equatorial magnetic field deviation is usually given by the Dst index. This is the hourly average of the deviation of $\mathrm{H}$ (horizontal) component of the magnetic field measured by several ground stations in mid to low-latitudes. Dst $=0$ means no deviation from the quiet condition, and Dst $\leq-50 \mathrm{nT}$ means magnetic storms $[3,4]$. We have analyzed the events represented by maximum Dst decrease and selected by using the selection procedure of Loewe and Prolss [9]. A list of magnetic storms based on the Dst indices provided by the World Data Center for Geomagnetism, Kyoto, Japan through its world wide web ( and also from the omni web data source maintained by National Geophysical Data Center (NGDC) : (http://www. ngdc. noaa. gov/ stp/SOLAR/ ftpsatenvir. html) is being compiled for this study for the period 1996-2007. As the study period refers to the interval solar cycle 23 . We have used the Omni Web Data Results (www. omniweb.gsfc. nasa. gov) [10-12, 25-26]. For the above tasks the data is collected from the online sources provided by the World Data Center through its web (National Oceanic and Atmospheric Administration (NOAA): http:// www. noaa. gov/), Geostationary Operational Environmental Satellite (GOES) : (http:// goes. ngdc. noaa.gov/) and also the flares observed by PRL's “Solar X-ray Spectrometer (SOXS) were also used, the data of which is obtained from the SOXS's homepage website URL link at http:// www.prl. res. in/ soxs-data/ The link provides solar data of various activities, Earth's atmosphere and geo-space disturbances and data for other planets. The SOXS was launched onboard GSAT-2 Indian spacecraft in 2003 using GSLV-D2 rocket. The SOXS provides solar flare observations in X-ray waveband in the energy range of 4-56 keV. [27-30].

\section{Methodology of the AnAlysis}

We deal with the sudden, sharp and short-lived depressions in the magnetospheric ring current and simultaneous solar parameters to understand the relationship. It is known that the intensity of solar parameters (e.g., solar flare, SEP flux etc.) is registered by satellite at the geostationary orbit in the near Earth space whereas the magnetic field variation and ring current depressions are recorded by a network of observatories well located all over the world. The data beig compiled the world data center Kyoto and distributed to world scientific community.Ultimately, there is a time-delay between the registration of Dst and the registration of solar parameters. To find out the time-delay, we availed cross-correlation analysis. We used cross-correlation function because it shows correlations at different points of two waveforms thereby exhibiting all correlations between every two signals of the waveforms. The time-delay is then calculated as the time-length with respect to the specific wave point where the highest correlation is found.

Cross-correlation analysis provides correlations between data of two time series or waveforms. The observations of one data series are correlated with the observations of another data series at various lags and leads. Cross-correlations help identify variables which are leading indicators of other variables or how much one variable is predicted to change in relation with the other variable. The cross-correlation test of two time-series data sets involves many calculations of the coefficient (r) by time-shifting the one data set relative to the other data set. Each shift is called a 'lag' and the lag time is simply the sampling period of the two time-series data sets. A typical cross-correlation graph shows enough lags in both negative and positive directions to show the cyclical relationship of the two sets of data. Detailed explanations on cross-correlation theorem, mathematical expressions and computation process can be studied in several books and papers e.g., Kuglin and Hines, 1975; Goshtasby et al., 1984; Mitra and Kaiser, 1993; Lewis, 1995; Qureshi, 2003 [ 32-36].

\section{OBSERVATIONS}

The 11-year solar activity cycle has been studied for a very long time Sunspot data is known to possibly data back to the ancient Chinese astronomers however the sun-earth connection is relatively new. The fact that the solar activity is directly related to space weather and geomagnetic activity does rise and fall along with the solar activity, In the whole period (1996-2007) of solar cycle-23, Solar cycle contains one maximum peak, where sunspot number is maximum and the period of that peak is termed as solar maximum activity phase [37-39]. So, the maximum phase of solar cycle-23 has been measured during the year 2000 whereas the periods 1996-99 and 2001-07 are the periods of minimum phase of solar activity which shown in figure 1 . 


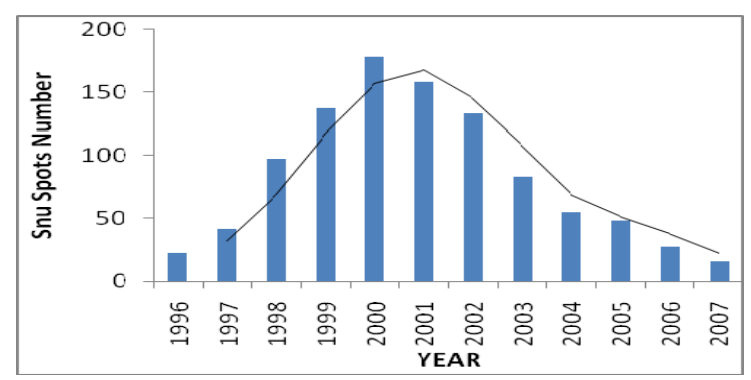

Fig. 1. Average number of sun spots per year during 1996-2007.

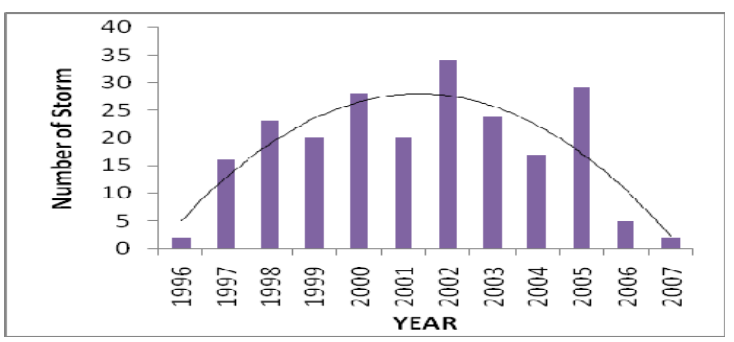

Fig. 2. The total number of storm days per year during 1996-2007.

In present study, we have used Dst data, that record the number and severity of geomagnetic storms during solar cycle-23. We have plotted this data and we can give answer several question having to do with how often geomagnetic storms occur during the year, and the frequency of their severity. Firstly we have investigated 220 geomagnetic storms with Dst $\leq-50 \mathrm{nT}$, are occurred during 1996 to 2007 . During this period 220 geomagnetic storms have been found to satisfy selection criteria and will compare with sunspots cycle-23.

Figure 1 gives the averaged sunspot number for that year and figure 2 gives the number of days in which geomagnetic storms were more severe than Dst $<-50 \mathrm{nT}$ and shown are the total number of storm days per year. Here, we have analyzed about 220 geomagnetic storm occurred during period 1996-2007. The number of geomagnetic storm observed in each year along with the sunspot number is shown in figure 1 and figure 2. From figure 2 it is evident that in the year 1996 (Solar minimum year) only 2 geomagnetic storm have occurred. It is also found that maximum numbers of geomagnetic storm have occurred in year 2002, while year 2000 is the maxima of the solar cycle-23, the year 2007 represents minimum sunspot activity during the descending phase of solar cycle-23, in the year 2003 and 2005, the large numbers of geomagnetic storm have occurred.

However the exact time span and intensity are up for dispute our data in figure 2 shows that the second descending phase geomagnetic peak occurs only 18-24 months after solar maximum. This data also shows that the descending phase peak seems to be larger, which means more activity occurs during that period, than the ascending phase peak. Therefore no significant correlation between the maximum and minimum phases of solar cycle and yearly occurrence of geomagnetic storm has been found.

Based on 220 geomagnetic storms (Dst magnitude <-50nT) occurred during year 1996 to 2007, we have classified geomagnetic storms with respect to their Dst magnitude in four categories according to Loewe and Prolss [9], a geomagnetic storm can be weak (Dst $>-50 \mathrm{nT})$, moderate $(-100 \mathrm{nT}<\mathrm{Dst} \leq-50 \mathrm{nT})$, intense (Dst $\leq-100 \mathrm{nT})$, and severe (Dst $\leq-200 \mathrm{nT}$ ).
TABLE I. SHOWS THE OCCURRENCE OF THE GEOMAGNETIC STORMS WiTH Their Classification as PER YeAR.

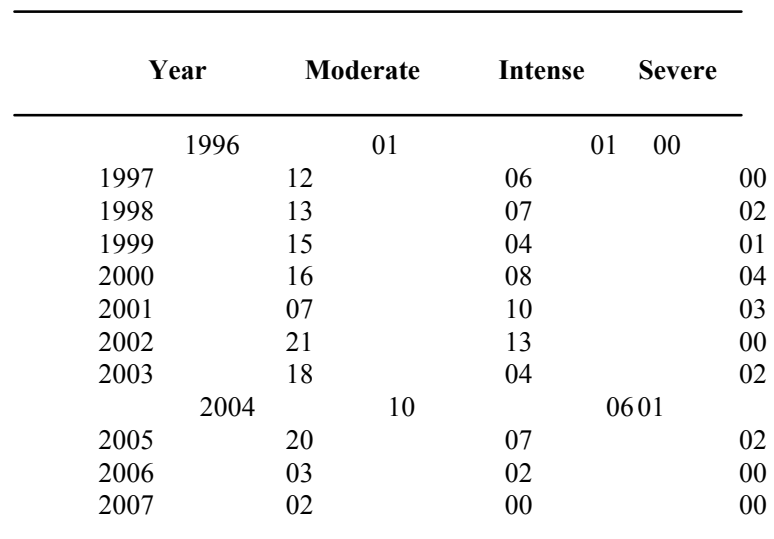

Table 1 shows the occurrence of the geomagnetic storms with their classification as per year. Under the selection criteria 138 moderate geomagnetic storms, 67 intense geomagnetic storms and 15 severe geomagnetic storms have been observed.

\section{RESUlT AND DISCUSSION}

Schwenn (2006) reviewed in details the solar processes associated with space weather phenomena Dameris and Pawson (2002) reviewed the effects of solar activity on the middle atmosphere and found controversial results since no clear physical mechanism existed to explain the interactions. Further, it is extremely difficult to isolate any solar-induced variability, since the dominant influence on the middle atmosphere appears to be tropospheric forcing. Experiments with two types of numerical model used to examine the atmospheric response to changes in solar forcing were reviewed by them. Firstly, mechanistic-model simulations of the solar-induced 27- and 13-day oscillations show that weak perturbations generated in the upper stratosphere can lead to detectable oscillations in the lower atmosphere. Secondly, a general circulation model shows that the modulation of the middle atmosphere dynamics by solar activity and the equatorial quasi-biennial oscillation is feasible. There are limitations to the studies. Thus, it is utmost important to improve our current understanding on the identifying the solar disturbances and their interaction with atmosphere as a function of altitude. This will lead to understand the coupling process among magnetosphere, ionosphere and thermosphere of the Earth.

Electromagnetic fields and currents connect various regions of the Earth's near space environment extending up to the magnetopause. Realization of this fact has lead to the concept of Global Electric Circuit (GEC) to describe the electromagnetic environment of the Earth's atmosphere. Solar wind - magnetosphere - ionosphere coupling forms a vital component of GEC. Magnetospheric sub-storms represent a global interaction between the solar wind, the magnetosphere, and the ionosphere. Lakhina (1994) reviewed the solar wind - magnetosphere- ionosphere coupling processes with emphasis on the nonlinear particle dynamics in the magnetotail [3]. Those aspects of the sub-storm processes which involve the chaotic dynamics are 
highlighted. Various methods based on nonlinear particle dynamics, linear prediction filtering techniques, phase space reconstruction techniques, and dynamical analogue models of geomagnetic activity are reviewed. It is shown that the solar wind - magnetosphere - ionosphere system behaves as a strongly coupled nonlinear dynamical system which could be driven from regular to chaotic behavior with low dimensionality when the solar wind forcing is strong enough.

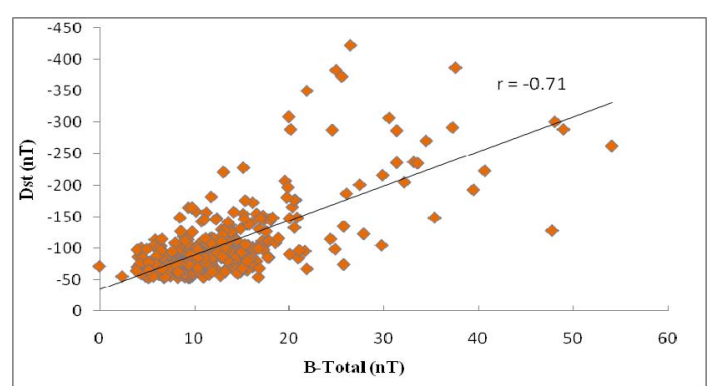

Fig. 3. presents the Interplanetary magnetic field versus the maximum of negative Dst.

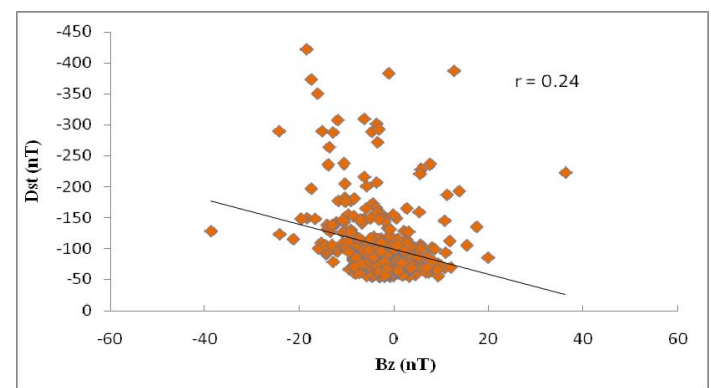

Fig. 4. presents the maximum of interplanetary negative Bz (southward) versus the maximum of negative Dst.

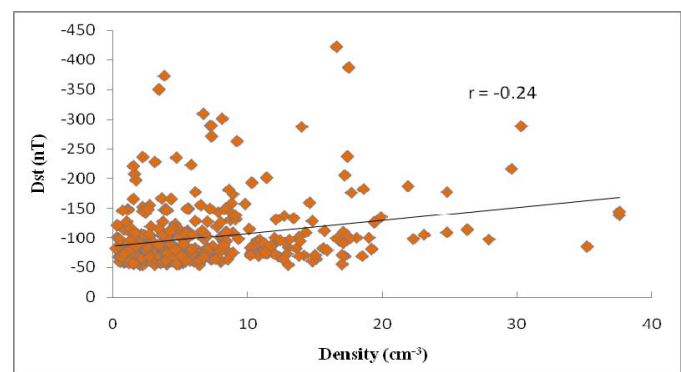

Fig. 5. shows the peak proton density versus the maximum Dst (negative)

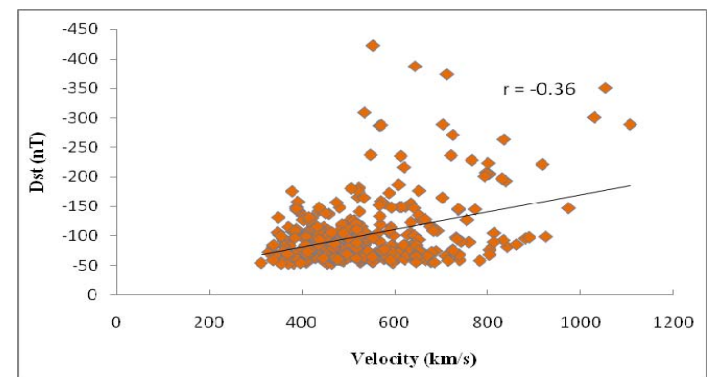

Fig. 6. presents mamimum values reached by the solar wind speed V versus negative Dst (max.)

Fig. 3 presents the Interplanetary magnetic field versus the maximum of negative Dst. Statistically, the occurrence of more intense geomagnetic storms (negative Dst magnitudes $\sim 250 \mathrm{nT}$ or less) is lower ( $\sim 10 \%$ of the storms considered). In this figure, a linear correlation between $B_{\text {total }}$ and Dst can be seen, that is, the strength of the geomagnetic storm is strongly dependent on the total magnetic field $\mathrm{B}_{\text {total }}$. The correlation coefficient has been found to be reasonably high (-0.71).

Figure 4 presents the maximum of interplanetary negative $\mathrm{Bz}$ (southward) versus the maximum of negative Dst. In this figure, a linear correlation between $\mathrm{Bz}$ and Dst can be seen, that is, according to previous studied the strength of the geomagnetic storm is strongly dependent on the southward component Bz. But in present study the correlation coefficient has been found to be low (0.24). This result may be obvious Solar wind Southward magnetic field component Bz has significant growth mainly during (or before) the initial phase of geomagnetic storm (not during the main phase, tested here). Absence of high linear correlation between density and Dst during the main phase does not mean that solar wind Southward magnetic field component $\mathrm{Bz}$ is not a geo-effective parameter, which is considered above. Studies shows the delay between the peak negative Dst and the negative $\mathrm{Bz}$ (at the time of Dst peak).

Figure 5 shows the peak proton density versus the maximum Dst (negative). No definite relationship between both these parameters is found. It can be seen the greater intensity geomagnetic storms are not necessarily associated with greater values of solar wind density. This means that there is a high probability that intensity of a geomagnetic storm is not determined by the increased density. The correlation coefficient between both these parameters is -0.24 .

Figure 6 presents maximum values reached by the solar wind speed $\mathrm{V}$ versus negative Dst (max.). The scatter is larger, with a wide range of velocities varying between 400 and $900 \mathrm{kms}^{-1}$. The more intense geomagnetic storms (peak Dst $<-350 \mathrm{nT}$ ) are not associated with greater values of solar wind velocities. The correlation coefficient between $\mathrm{V}$ and peak Dst has been found to be -0.39 .

\section{CONCLUSION}

The maximum phase of solar cycle- 23 has been measured during the year 2000 whereas the periods 1996-99 and 2001-07 are the periods of minimum phase of solar activity. Which clearly follow the phase of sunspots cycle. It is evident that in the year 1996 (solar minimum year) only 2 geomagnetic storm have occurred. It is also found that maximum number of geomagnetic storm have occurred in year 2002 while year 2000 is the maxima of the solar cycle-23, the year 2007 represent minimum sunspot activity during the descending phase of solar cycle-23. The largest geomagnetic storm of solar cycle-23 occurred on 20 November 2003, with a Dst index of $-472 \mathrm{nT}$ and the large numbers of geomagnetic storm have occurred in the year 2003 and 2005, which do not exactly follow the phase of solar cycle and show complex behavior. It is believed that the majority of intense geomagnetic storm occur during the maximum phase of sunspot cycle because many solar active region appear during this time while a few of the geomagnetic storms are observed during the minimum phase of sunspot cycle, which do not exactly follow the phase of solar cycle and show complex behavior.

The present paper has considered the peak values of the various parameters which are further correlated with the peak depression in the geo-magnetic perturbed conditions. It is 
widely recognized that the solar and interplanetary causes produce geomagnetic disturbances. In present study, a linear correlation between $\mathrm{B}_{\text {total }}$ and Dst can be seen, that is, the strength of the geomagnetic storm is strongly dependent on the total magnetic field $\mathrm{B}_{\text {total }}$. The correlation coefficient has been found to be reasonably high (-0.71).

According to previous studied the strength of the geomagnetic storm is strongly dependent on the southward component Bz. But in present study the correlation coefficient has been found to be low (0.24). This result may be obvious Solar wind Southward magnetic field component Bz has significant growth mainly during (or before) the initial phase of geomagnetic storm (not during the main phase, tested here). Thus, in this study period had something special which need to be understood, Bz is not essentially peak at the time of dst peak value. This shows time delay between $\mathrm{Bz}$ and Dst peak.

It has been verified that geomagnetic storm intensity is correlated well with the total magnetic field of IMF better than southward component Bz of the IMF, density and solar wind velocity. However, it is clear that present analysis should be considered preliminary, mainly because of the uncertainty in time delay, which should be investigated in detail for prediction purposes.

\section{ACKNOWLEDGMENT}

This work is financed by UGC, India through its research scheme: Data of the space satellite of NGDC, CDAW of NASA and data of geophysical parameters provided by World Data Center, Kyoto Japan and also the solar flares data observed by Solar X-ray Spectrometer (SOXS); PRL / ISROs first space mission onboard GSAT -2, have been utilized. The author thanks the PI's for providing these satellite data. SCK and BVS are grateful to Prof. Rajmal Jain, PI-SOXS and SWEP: Chandrayaan-2 mission and Sr. Scientist, PRL, Ahmadabad for his encouragement and helpful discussions during their visit to PRL. K A Firoz thanks 'Development of Korean Space Weather Center' of KASI for providing the necessary support. We thank the anonymous adjudicator for constructive comments and suggestions that developed the manuscript. We also thank the anonymous reviewers for their comments.

\section{REFERENCES}

[1] S.I. Akasofu, "Solar-wind disturbances and the solar wind-magnetosphere energy coupling function," Space Sci. Rev., 34, 173-183, 1983

[2] J. A. Joselyn and P. S. McIntosh, "Disappearing solar filaments: A useful predictor of geomagnetic activity,” J. Geophys. Res., 86, 4555, 1981

[3] Lakhina G.S., "solar wind-magnetosphere-ionosphere coupling and chaotic dynamics", Surveys in Geophysics, Vol 15, No.6/Nov, pp 703-754 DOI: 10.1007/ BF00666091, 1994.

[4] W. D. Gonzalez, J. A. Joselyn, Y. Kamide, H. W. Kroehl, G. Rostoker, B. T. Tsurutani, V. M. Vasyliunas, "What is A Geomagmetic Storm?,” J. Geophys. Res., 99, 5771-5792, 1994

[5] Kaushik, S.C. and Shrivastava, P.K., Influence of magnetic clouds on interplanetary features, Indian Journal of Physics, 74 B (2), 2000, $159-162$.

[6] Kaushik, S. C. and Shriastava, P.K., An investigation of two types of interplanetary transient disturbances and their effects on cosmic ray intensity in relation with solar wind plasma parameters, Indian Journal of Radio and Space Physics, 28,1999,203-210.
[7] Shea, M. A and D. F. Smart (1990), A summary of major solar events, solar physics, 127, 297-320

[8] Shriastava, P.K., and Agarwal S P, ; Proc. Basic Plasma Processes on the Sun, Kluwar Academy Press, Holland, p.259, 1990.

[9] Kuznetsov V.V., Plotkin V.V., Nesterova G.V., Nesterova I.I., "Universal variation of the F2-layer critical frequency and Solar activity", Earth Planets Space, Vol 50, pp 57-61, 1998.

[10] C. A. Loewe and G.W. Prolss, "Classification and mean behavior of magnetic storms," J. Geophys. Res. 102, 14209, 1997

[11] Gopalswamy, N., Yashiro, S., Krucker, S., Stenborg, G., Howard, R.A. Intensity Variation of large solar energetic particle events associated with coronal mass ejections. J. Geophys. Res. 109, A12105 (2004). doi:10.1029/2004JA010602.

[12] R. A. Howard, N. R. Sheeley Jr., M. J. Koomen and D. J. Michels, "Coronal Mass Ejections, 1979-1981," J. Geophys. Res., 90, $8173-8191,1985$

[13] G. Zhang and L.F. Burlaga, "Magnetic clouds geomagnetic disturbances, and cosmic ray decreases,” J. Geophys. Res., 93, 2511, 1988

[14] Kaushik, S. C. and Shriastava, P.K., Effects of interplanetary transients disturbances on cosmic ray intensity, Bulletin of Astronomical Society of India, 27,1999,85-90.

[15] Kaushik, S.C., A study of intense geomagnetic storms and their associated solar and interplanetary causes, Coronal and stellar mass ejections, Proc. IAU Symposium, Cambridge University Press, 2005, p. 454.

[16] Mishra S.K., Tiwari D.P. and S. C. Kaushik, Forbush decrease event and associated geomagnetic field variation during space radiation storm, International Journal of Modern Physics - A, 20, No. 29, 2005, 6717.

[17] Firoz, K.A., Kumar Phani, D.V., Cho, K.-S.: On the relationship of CRI with solar, interplanetary, and geophysical parameters. Astrophys. Space Sci. 325(2), 185-193 (2009)

[18] Firoz, K.A., Kudela, K.: Diurnal variation of cosmic rays and low energy particle fluxes. In:WDS Prague, Part II, ISBN 978-80-7378024-1, pp. 106-110 (2007)

[19] Gopalswamy, N., Xie, H., Yashiro, S., Usoskin, I.: Coronal mass ejections and ground level enhancements. In: 29th Int. Cosmic Ray Conf., vol. 1, pp. 169-172 (2005) D. F. Webb and R. A. Howard, "The solar cycle variation of coronal mass ejections and the solar wind mass flux," J. Geophys. Res., 99, 4201, 1994

[20] Sabbah, I. (2000): The influence of transient solar wind events on the CRI modulation, Canadian Journal of Physics, 78, 293-302

[21] Firoz, K.A., Cho, K.-S., Hwang, J., Phani Kumar, D.V., Lee, J.J.,Oh, S.Y., Kaushik, S.C., Kudela, K., Rybanský, M., Dorman, L.I.: Characteristics of ground level enhancement associated solar flare, coronal mass ejection, and solar energetic particle. J. Geophys. Res.(2010). doi:10.1029/2009JA015023

[22] I. Sabbah, "The role of interplanetary magnetic field and solar wind in modulating both galactic cosmic rays and geomagnetic activity," Geophys. Res., Lett., 27, 13, 2000

[23] Aschwanden, M. J. (2010), GeV Particle Acceleration in Solar Flares and Ground Level Enhancement (GLE) Events, Space Sci. Rev., arXiv:1005.0029v1 [Astro-ph.SR]

[24] Belov, A.V.: Properties of solar X-ray flares and proton event forecasting, Advances in Space Research, 43, 467 - 473 (2009)

[25] J. H. King, "Interplanetary Medium Data Book," NSSDS, WDC-A, Maryland, 1997

[26] J. H. King and N. E. Papitashvili, "Interplanetray Medium Data Book," NSSDS, WDC-A, 1998S.

[27] Jain R., Dave H., Shah A.B., Vadher N.M., Shah V.M., Ubale G.P., Manian K.B.S., Solanki C.M., Shah K.J., Kumar S., Kayasth S.L., Patel V.D., Trivedi J.J., and Deshpande M.R., "Solar X-ray Spectrometer (SOXS) Mission onboard GSAT-2 Indian Spacecraft: The Low Energy Payload", Solar Phys., 227, pp 89-122, 2005.

[28] Jain R., Deshpande M.R., Dave H.M., and 16 other coauthors, "Technical Document-GSAT-2 Spacecraft, Prelimnary Design Review (PDR), Document for Solar X-ray Spectrometer, ISRO-ISAC-GSAT-2-PR-0155, Bangalore", Published by Indian Space Research Organisation.

[29] Jain R., Joshi V., Kayasth S.L., Dave H., Deshpande M., "Solar X-ray Spectrometer (SOXS) mission-Low Energy Payload-First results", J. Astrophys. Astron., Vol 27, pp 175-192, 2006.

[30] Jain R., Malini A. and Sharanma R., “ X-ray emission from flares”, J. Asrtrophys. Astr, Vol 29, pp 125-145, 2008.

[31] Jain R., Rao A.R., Deshpande M.R., Dwivedi B.N., Mnoharan P.K., Seetha S., Vahia M.N., Vats H.O. and Venkatkrishnan P., "Science from Solar X-ray Spectrometer Low Energy Detector (SLD)- Proposed 
payload onboard Indian Satellite", Bull. Astr. Soc. India, 28, 121-122, $2000 \mathrm{a}$.

[32] Kuglin, C. and Hines, D.; The phase correlation Image Alignment Method, Proc. Intl. Conf. Cybernetics and Socity, 183-165, 1975;

[33] Goshtasby, A. S, Gage, S.H., Bartholic, , J. F. , A Two Stage CrossCorrelation Approach to Template Matching. IEEE Trans. Pattern Analysis and Machine Intelligence, 6 (3), 374-378; 1984;

[34] Mitra, S. K. and Kaiser, J. F.; Handbook for Digital Signal Processing, New York, Wiley Eastern, 1993;

[35] Lewis, J. P.1 ; Fast Template Matching, Vision Interface, 120-123, 1995;

[36] Qureshi, Shehrzad (2003), "Cross-Correlation and Matched Filters", Dr Dobb's Journal, Edition of April 2003, page: 52-56.

[37] Kumar S., Mishra S.D., Vijay S.K. and Gwal A.K., "Day to day variability in ionospheric electron content at low latitudes during the minima of 20th and 21st solar cycle", Acta Geophysica Geodaetica Hungarica, Vol 32, pp 293-299, 1997.

[38] Smart, D. F. and M. A. Shea (1989), Solar proton events during the past three solar cycles, J. Spacecraft Rockets, 26, 403-415

[39] Asai, A., H. Nakajima, and M. Shimojo, (2006), Preflare Nonthermal Emission Observed in Microwaves and Hard X-rays, Astron. Soc. Japan, 58, L1-L5

[40] D. A. Couzens and J. H. King, "Interplanetary Medium Data Book," NSSDS, WDC-A, Maryland, Suppl. 3, 1986

[41] Kaushik, S.C., Geomagnetic and ionospheric responses to changes in IMF, Proc. IAU Asia Pacific Regional IAU Meeting, eds. Premana Premdasa and Mehsana, Cambridge University Press. 2005, p.77

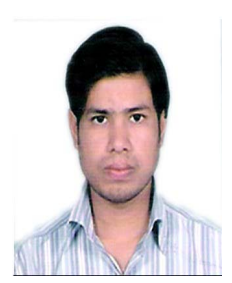

Rathor Born on January 5, 1984, he did his M.Sc and M. Phil. from School of Studies in Physics, Jiwaji University, Gwalior in 2006 and 2008 respectively. At present he is working for his Ph.D. Program. His main interest is solar wind transients, modulation effects and geo-effectiveness. He has published 05 research papers and attended 10 national and international research conferences.

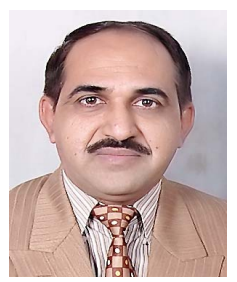

Kaushik born on March 10, 1968, and did his M.Sc and M. Phil. from fro $\mathrm{m}$ Institute of Advanced Studies, Meerut University. He obtained his Ph.D. in 2002. He has completed 04 research projects and recipient of several awards and fellowships. $\mathrm{He}$ has visited more than 15 countries and gave invited /oral talks. He is the member of various scientific bodies. His main interest is in Interplanetary Plasma transients, geomagnetic effects and cosmic rays.

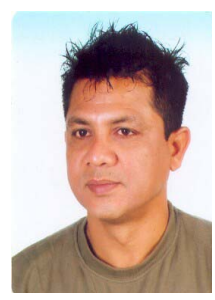

Firoz born in Bangladesh and obtained his Ph.D. from University of Pavol Jozef Šafarik, Košice, Slovakia. Later as a Post Doctor he joined the Space Weather Research Group, Korea Astronomy and Space Science Institute, Daejeon, South Korea. His main interest is in cosmic ray studies, The Ground Level Enhancement effects and Forbush Effects. He has published more than 15 papers in highly reputed International Journals and attended $\sim 10$ research conferences abroad.He has published / presented 115 research papers in International/ National journals and conferences

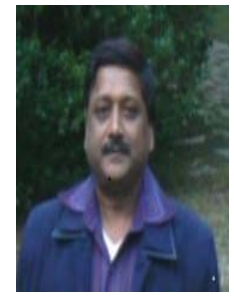

Gupta did his Ph.D. from Bhopal University in 1991. He has been the recipient of various research fellowships from UGC / DST. He has six years of post-doctoral research experience. He has been a visitor to Abdus Salam International Centre for Theoretical Physics, Trieste (Italy) as scientist. He has completed one major research project from UGC, New Delhi and one more project from DST, New Delhi is in hand..

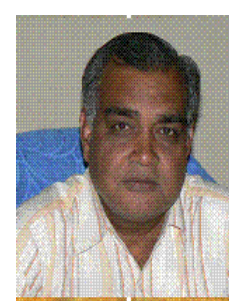

Shrivastava did his M.Sc. from Jiwaji University Gwalior, and received CSIR Fellowship for doing $\mathrm{Ph}$.D. Presently he is Professor of Physics in Jiwaji University. He is member of various scientific bodies / societies and published over 30 research papers in International journals and presented more than 50 research papers in conferences. He has completed research projects funded by UGC and MPCOST. He has a vast experience of Research and administration

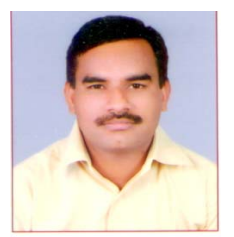

Parashar did his M. Sc. and M.C.A. from Jiwaji University, Gwalior and at present working for his $\mathrm{Ph} . \mathrm{D}$. Program. His main interest is in developing the software tools useful for astronomical studies. He is also interested in solar wind modulation effects and potentially geo-effective phenomena. He published 02 research papers and attended 06 research conferences

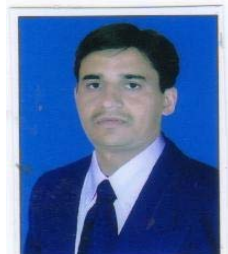

Bhaudoriya Born on July 15, 1976 in district Morena (MP), he did his M.Sc. from Jiwaji University, Gwalior and at present working for his $\mathrm{Ph} . \mathrm{D}$. Program. He is also a visiting faculty in YVS College, Gwalior. His main interest is solar wind transients and cosmic ray modulation effects. He has published 02 research papers and attended 06 research conferences 